\title{
La línea estratégica de mitigación del riesgo en los planes de manejo de los sitios de Patrimonio Mundial: propuesta de un sistema de indicadores y su aplicación en Italia
}

The Strategic Approach for Risk Mitigation in the Management Plans of World Heritage Sites: A Proposal for an Indicator System and its Application in Italy

\section{Daniela Andrea Díaz Fuentes}

Università degli Studi della Basilicata (Unibas), Italia

daniela.diazfue@gmail.com

\section{Resumen}

En los últimos decenios, los terremotos han dañado una gran cantidad de patrimonio cultural en Italia, país que cuenta con el mayor número de sitios inscritos en la Lista del Patrimonio Mundial. Ante los requerimientos de la Organización de las Naciones Unidas para la Educación, la Ciencia y la Cultura (UNESCO, por sus siglas en inglés) de desarrollar planes de manejo y prevenir el riesgo de catástrofes, varios sitios en ese y otros países han manifestado la importancia de incluir a la evaluación de los riesgos dentro de esos instrumentos de planificación. Sin embargo, hasta la fecha no se ha definido una línea estratégica específica de mitigación del riesgo asociada a indicadores y mapas con los que orientar la programación de acciones. La presente INVESTIGACIÓN analiza diversas propuestas metodológicas para la planificación estratégica del patrimonio cultural con el objeto de incorporar la línea de mitigación del riesgo mediante un sistema de indicadores cualitativos y cuantitativos que se enfoquen al análisis tanto de las amenazas a escala territorial como de la vulnerabilidad sísmica de edificios de mampostería de piedra, ladrillo y adobe. Finalmente, estas aproximaciones se aplican en el sitio UNESCO I Sassi e il parco delle chiese rupestri di Matera (Los Sassi y el conjunto de iglesias rupestres de Matera), Italia.

\section{Palabras clave}

evaluación del riesgo; planes de manejo; vulnerabilidad; amenazas; sitios de Patrimonio Mundial; Italia

\section{Abstract}

In recent decades, earthquakes have damaged several cultural heritages in Italy, the country with the largest concentration of sites inscribed on the World Heritage List. Taking into consideration the requirements of the United Nations Educational, Scientific and Cultural Organization (UNESCO) to develop management and prevention plans for the risk of catastrophes, several sites in Italy and other countries have expressed the importance of including risk assessment into these planning instruments. Yet, to this day, no specific strategic line for the mitigation of risks, associated with indicators and maps have been developed in order to program actions. This RESEARCH analyzes dif- 
ferent methodological proposals for the strategic planning of heritage sites, in order to incorporate a risk mitigation approach through a system of qualitative and quantitative indicators that are focused on the analysis of both threats at a territorial scale and the seismic vulnerability of rock, brick, and adobe masonry buildings. Finally, these approaches are applied on the UNESCO site I Sassi e il parco delle chiese rupestri di Matera (The Sassi and the Park of the Rupestrian Churches of Matera), Italy.

\section{Keywords}

risk assessment; management plans; vulnerability; threats;

World Heritage sites; Italy

\section{Introducción}

talia, país que cuenta con el mayor número de sitios Patrimonio de la Humanidad, con un total de 51 inscritos en la lista correspondiente (UNESCO 2017), ha sufrido los efectos de varios terremotos en los últimos decenios. ${ }^{1}$ El acontecido en la región Umbria y Marche en 1997 dañó más de 10000 edificios —algunos los destruyó-, entre ellos, muchas iglesias medievales, palacios y construcciones de vivienda históricas (ICOMOS 2000), mientras que el del año 2002 en la región de Molise y Puglia, si bien no ha sido uno de los más intensos en términos de magnitud -5.74 Mw-, deterioró, según los registros (Podestà et al. 2010:332), 586 edificios monumentales, $75 \%$ de los cuales correspondió a iglesias, $15 \%$ a palacios y $4 \%$ a conventos y torres.

Pese a lo anterior y a la alta sismicidad del territorio italiano, en el año 2012 sólo 25 sitios de patrimonio mundial contaban con un plan de manejo, 6 de los cuales estaban en etapa de ejecución (Badia 2012:52). Por lo tanto, una gran mayoría de estos lugares patrimoniales carecía de planificación estratégica para abordar su resiliencia ante los cambios. Es de notar que se ha recalcado la importancia de la evaluación continua para el manejo del cambio en Managing Cultural World Heritage (UNESCO/ICCROM/ICOMOS/IUCN 2013), manual que subrayó: "si se requieren cambios — tanto para mejorar el sistema de manejo como para asegurar que responda a nuevas necesidades-, la evaluación por sí misma deberá establecer cómo introducirlos" (UNESCO/ICCROM/ICOMOS/ IUCN 2013:54, trad. de la autora). Por ejemplo, puede ser necesario hacer cambios ante una situación catastrófica al momento en que el Comité de Patrimonio Mundial inscribe el bien cultural en la Lista del Patrimonio Mundial en Peligro² (UNESCO/ICCROM/ICOMOS/IUCN 2013:144). Asi-

\footnotetext{
${ }^{1}$ Friuli en 1976, Irpinia en 1980, Umbria y Marche en 1997, Lacio en 1999, Toscana en 1995, Piamonte en el 2000, Molise en el 2002, Aquila en el 2009, Emilia-Romagna en el 2012 y Centro Italia en el 2016 (INGV 2017).

${ }^{2}$ Con base en el informe sobre el estado de conservación, en el asesoramiento de los órganos consultivos y en la consulta con los Estados Partes, el comité puede incluir bienes en la Lista del Patrimonio Mundial
}

mismo, es relevante subrayar que, con el fin de controlar los efectos de las catástrofes asociadas a fenómenos naturales o antrópicos, dicho comité recomienda a los Estados Partes incluir la prevención del riesgo en las líneas estratégicas de los planes de manejo de los sitios de Patrimonio Mundial (UNESCO-WHC 2015:23). También se requiere que tanto en el desarrollo como en la puesta en práctica y el monitoreo de las líneas estratégicas se definan indicadores cuantitativos y cualitativos, "ya que esta documentación es necesaria para el seguimiento de la sostenibilidad de los sitios" (Labadi 2015:154, trad. de la autora).

En la actualidad se cuenta con varios esquemas metodológicos o modelos de planificación estratégica, desarrollados en varios lugares del mundo para sitios patrimoniales (por ejemplo, Demas 2002; Medina-González 2006; Johnston 2010), todos ellos basados en The Australia ICOMOS Charter for Places of Cultural Significance (Australia ICOMOS 2013 [1979]), mejor conocida como la Carta de Burra. La presente INVESTIGACIÓN analiza algunos de esos esquemas con el objeto de alentar que se incorpore en los planes de manejo una línea estratégica de mitigación del riesgo, y propone un sistema de indicadores cualitativos y cuantitativos para la evaluación de su sostenibilidad. Éstos se enfocan, principalmente, en el análisis tanto de las amenazas a escala territorial como de la vulnerabilidad sísmica de edificios de mampostería de piedra, ladrillo y adobe. Finalmente, la propuesta se aplica en un ejercicio de evaluación a un caso de estudio: el sitio inscrito en la Lista de Patrimonio Mundial de la UNESCO I Sassi e il parco delle chiese rupestri di Matera (Los Sassi y el conjunto de iglesias rupestres de Matera) (Colonna y Fiore 2014), mejor conocido como Sassi di Matera, en Italia.

\section{Breve revisión de esquemas metodológicos o modelos de planificación estratégica patrimonial}

James Kerr (2013 [1996]) define el instrumento denominado plan de manejo para la conservación de los sitios de relevancia cultural europea como "un documento que establece lo que es significativo en un lugar $y$, en consecuencia, qué políticas son apropiadas para permitir que se mantenga esa significación en su desarrollo y uso futuro. En la mayoría de los lugares, aborda el manejo del cambio." (Kerr 2013 [1996]:1, trad. de la autora). El modelo de Kerr (2013 [1996]) estableció un proceso lógico basado en el significado (Figura 1), el cual comienza con la comprensión del sitio mediante su descripción detallada y continúa con una evaluación de su relevancia y la definición de la Declaración de Significación; posteriormente, se propone analizar la condición física del sitio y los requerimientos o presiones externas para, finalmente,

en Peligro en virtud del artículo 11.4 de la Convención del Patrimonio Mundial (UNESCO 1972). 
ETAPA 1

Entender el sitio

Recolectar información (bibliográfica y física)

Coordinar y analizar la información

Evaluación y declaración de significado

ETAPA 2

Política de conservación y su implementación$$
\text { | }
$$

Recolección de información para el desarrollo de la política de conservación

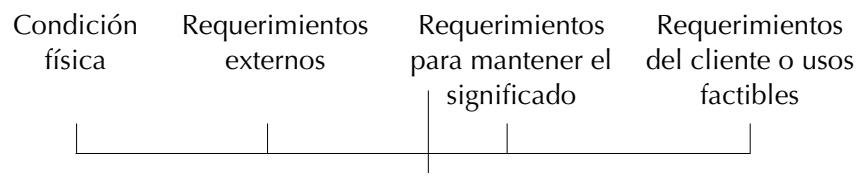

Desarrollo de la política de conservación

Establecer la política de conservación y las sucesivas estrategias y opciones para su implementación

FIGURA 1. Elementos básicos y secuencia en un plan de conservación (Fuente: Kerr 2013 [1996]:3).

desarrollar políticas que las afronten y también mejoren los aspectos positivos del sitio (Young 2002:61).

Varios autores (Demas 2002; Medina-González 2006; Johnston 2010) han propuesto variantes a las etapas de planificación definidas, primero, por la Carta de Burra (Australia ICOMOS 2013 [1979]) y desarrolladas, más tarde, por James Kerr (2013 [1996]), siempre considerando que el proceso de planificación presenta una progresión lógica, aunque no estrictamente lineal (Demas 2002:29). Estos modelos han incorporado el concepto de "manejo del cambio", al señalar la importancia de adaptar periódicamente, a través del monitoreo y la evaluación de indicadores, el plan de manejo. Sin embargo, un aspecto que se ha de subrayar es que sólo algunos de estos modelos han explicitado la noción de "gestión de los riesgos". En la tercera etapa del modelo de Johnston (2010:8) (denominada identificar problemas) se mencionan los riesgos derivados de fenómenos naturales - como las inundaciones, las tormentas y las sequías- y las amenazas antrópicas - como el robo, los incendios o el vandalismo-. Demas (2002:27) indica, asimismo, que pese a que no es posible resolver definitivamente los problemas derivados de las amenazas asociadas a los fenómenos naturales, el proceso de planificación debe abordarlos por razón de que "su impacto puede ser controlado o mitigado" (Demas 2002:27), lo cual posibilita que las decisiones para preservar los valores del sitio sean más sustentables.

Por su parte, la propuesta metodológica de MedinaGonzález (2006:61) en la etapa de planeación del futuro del sitio propone que se desarrollen políticas, estrategias y programas para el manejo y control de los desastres.
Ahora bien, con base en algunas obras de la bibliografía hasta aquí referida y en un proceso de consulta nacional a los encargados de los sitios UNESCO y de los sistemas turísticos locales, en el año 2004 el ministerio italiano para los Bienes y las Actividades Culturales y del Turismo (MIBACT, Ministero dei Beni e delle Attività Culturali e del Turismo) planteó un modelo de lineamientos para el desarrollo de los planes de manejo de los sitios inscritos como Patrimonio de la Humanidad, intitulado II modello del piano di gestione dei beni culturali iscritti alla lista del Patrimonio dell'Umanità. Linee Guida (MIBACT 2004). Éste propone un análisis integrado del sitio que identifica las fuerzas de modificación externas y los escenarios futuros, para desarrollar proyectos estratégicos y verificar "el cumplimiento de las metas mediante una serie de indicadores que permiten el monitoreo sistemático" (MIBACT 2004:21). En relación con la prevención y mitigación, el modelo (MIBACT 2004:15) señala que el nivel de riesgo depende de tres causas: el valor del bien cultural, las amenazas y la vulnerabilidad. Asimismo, señala que deben evaluarse los factores de daño intrínseco y extrínseco con el objeto de evidenciar, por ejemplo, la predominancia o frecuencia de un fenómeno determinado (MIBACT 2004:27), y recomienda consolidar la información con mapas de riesgos mediante bases de datos y un sistema de información geográfica (MIBACT 2004:50).

Los modelos de planificación para lograr el manejo del cambio propuestos por varios autores señalan la importancia de conocer la condición física del sitio y las presiones externas, para lo cual habría que basarse en procedimientos simplificados de evaluación del riesgo a partir de las variables que lo definen según /l modello italiano (MIBACT 2004): la vulnerabilidad, las amenazas y la exposición a éstas. Así, con la revisión de algunos procedimientos simplificados de evaluación del riesgo propuestos por otros autores se identificarían metodologías de diagnóstico del patrimonio cultural, y se definirían indicadores cuantitativos y cualitativos que podrían monitorearse sistemáticamente para la evaluación, adaptación y mejora del plan.

\section{El concepto del "riesgo" en los planes de manejo de los sitios de Patrimonio Mundial}

La Carta de Burra señala en su artículo $2^{\circ}$ que "los lugares de relevancia cultural deben salvaguardarse y no deben ponerse en riesgo o dejarse en un estado vulnerable" (Australia ICOMOS 2013 [1979]: 3, trad. de la autora). Asimismo, las Directrices prácticas (UNESCO 1972) recomiendan que "los Estados Partes incluyan la preparación para afrontar los riesgos como un elemento en los planes de manejo y líneas estratégicas de los sitios de Patrimonio Mundial" (UNESCO-WHC 2015:23, trad. de la autora). Por lo tanto, en la redacción de las políticas de planificación estratégica de un sitio Patrimonio Mundial parece ineludible considerar las vulnerabilidades y amenazas que lo 
ponen en riesgo. Desafortunadamente, las Directrices prácticas de la Carta de Burra (Australia ICOMOS 2013 [1979]) no clarifican la diferencia conceptual entre amenaza, vulnerabilidad y riesgo, ya que los tres se asocian a la amenaza antrópica tanto por falta de mantenimiento como por alteraciones negativas o demoliciones.

Sin embargo, el concepto de "riesgo" ya se ha utilizado para realizar la evaluación de la condición de los sitios tanto en la Carta del Rischio (Mapa del riesgo, ISCR 1992) en Italia, en MARS: The Monuments at Risk Survey of England, 1995: Main Report (Darvill y Fulton 1998), y en los informes periódicos de ICOMOS sobre los monumentos y sitios en peligro (2000).

Debido a la implementación de la Carta del Rischio (ISCR 1992) en Italia, el plan de manejo del sitio inscrito en la Lista del Patrimonio Mundial de la UNESCO Le città tardo barocche del Val di Noto (Las ciudades del Barroco tardío del Valle del Noto, Guido 2002) en Sicilia, identificó factores de amenaza ambiental en un sistema de información geográfica. ${ }^{3}$ Otro plan de manejo que se hace cargo de los riesgos es el del sitio The Old and New Towns of Edinburgh (Las ciudades antiguas y nuevas de Edinburgo, HS/CEC/EWT 2011), que a través de políticas locales aborda temas como: el cambio climático; el riesgo de incendio; la prevención de inundaciones, y las presiones del desarrollo descontrolado (HS/CEC/EWT 2011:64-66). Asimismo, el plan de manejo del sitio arqueológico de Stonehenge (Young et al. 2009) planteó la relevancia de desarrollar tanto una línea estratégica para identificar los riesgos potenciales derivados de las tormentas y del vandalismo como planes de emergencia (Young et al. 2009:70). Otros planes de manejo, como el de la Zona Arqueológica de Chichén Itzá, en México (INAH-Conaculta 2009), el de las Oficinas Salitreras Humberstone y Santa Laura, en Chile (CMN 2004), y el del sitio arqueológico de Joya de Cerén, en El Salvador (Castellanos et al. 2002), entre otros, también han resaltado la trascendencia de la evaluación, la prevención y el manejo del riesgo de desastres. Sin embargo, no se establecen indicadores medibles de amenazas y vulnerabilidad que permitan un monitoreo sistemático, como tampoco una metodología que haga posible realizar un diagnóstico global de los riesgos.

Un sitio de Patrimonio Mundial que presenta un alto riesgo asociado a fenómenos naturales es el Centro Histórico de la Ciudad de México, inscrito en la Lista del Patrimonio Mundial de la UNESCO en 1987, en conjunto con Xochimilco, asociando su valor universal excepcional a la transformación del paisaje (ACH-FCH CDMX 2011:5), por lo que su valor fundamental es también uno de sus principa-

\footnotetext{
${ }^{3}$ El Mapa del riesgo define tres tipos de amenazas. 1. Estática-estructural por fenómenos naturales: sísmicos, deslizamientos de ladera, dinámica de los litorales, volcánicos; 2. Ambiental, que genera la degradación de los materiales pétreos: índices de erosión, de ennegrecimiento y de estrés físico; 3. Antrópica: dinámicas de la densidad demográfica, presión turística, susceptibilidad al robo (Guido 2002:99-100).
}

les riesgos. Debido a su extensión y complejidad, el plan de manejo señala que es necesaria la retroalimentación entre la información contenida en los catálogos de patrimonio y los bancos de datos relativos al ordenamiento territorial y a los mapas de riesgos (ACH-FCH CDMX 2011:76). Sin embargo, probablemente por la falta de una metodología o de indicadores medibles para la evaluación de los riesgos, no se han analizado sistemáticamente todas las amenazas que podrían afectar el sitio, ni la vulnerabilidad sísmica de los edificios que, por las características del subsuelo lacustre, constituye otro riesgo importante.

Un sitio de Patrimonio Mundial emblemático por el precario estado de conservación de su arquitectura de tierra es la Zona Arqueológica de Chan Chan, en Perú, que fue inscrita en la lista de la UNESCO en 1986, y en el mismo año, incluida en la Lista del Patrimonio Mundial en Peligro (Castellanos 2001:110). El plan de manejo de este sitio no propone un mapa de riesgo, aunque el diagnóstico sí analiza sus vulnerabilidades y amenazas. Dentro de los indicadores de estas últimas se mencionan: eventos sísmicos; el fenómeno del niño, asociado a lluvias torrenciales, inundaciones y aluviones de lodo; la acción conjunta del sol, los vientos alisios que son húmedos y salinos, la humedad nocturna y las lluvias esporádicas; el deterioro por agentes biológicos, como el crecimiento de raíces y vegetación (Hoyle y Castellanos 1999:15-19), y factores relacionados con su manejo y con el contexto social, como el avance urbano y la producción agrícola e industrial (Castellanos 2001:109). Además, el proceso de planificación del sitio incluyó la zonificación como una etapa importante para afrontar las tareas a corto, mediano y largo plazos (Figura 2), y también para definir las intervenciones para disminuir las vulnerabilidades y mitigar las amenazas (Hoyle y Castellanos 1999:15).

Los planes de manejo actualmente instrumentados han reconocido la importancia de la evaluación, la prevención y el manejo del riesgo de desastres; sin embargo, una importante problemática en el abordaje del tema es que, a pesar de que existe un amplio acuerdo entre los administradores de los sitios respecto de las causas generales del deterioro de los bienes, no queda claro cómo medir los grados de amenaza y vulnerabilidad. Así, las estrategias y proyectos concretos de los planes han surgido para resolver factores de deterioro caso por caso, sin una metodología de diagnóstico global que prevea indicadores medibles de amenazas y vulnerabilidad, lo cual no redunda en el desarrollo de una zonificación en mapas de riesgos y un monitoreo sistemático. La definición de un sistema de indicadores, organizado en una base de datos integrada y con una zonificación coordinada con los instrumentos de planificación territorial darían la posibilidad de direccionar estudios e investigaciones específicos; realizar diagnósticos, al superponer zonificaciones de amenazas con inmuebles vulnerables, y priorizar el monitoreo y los proyectos de conservación preventiva. 


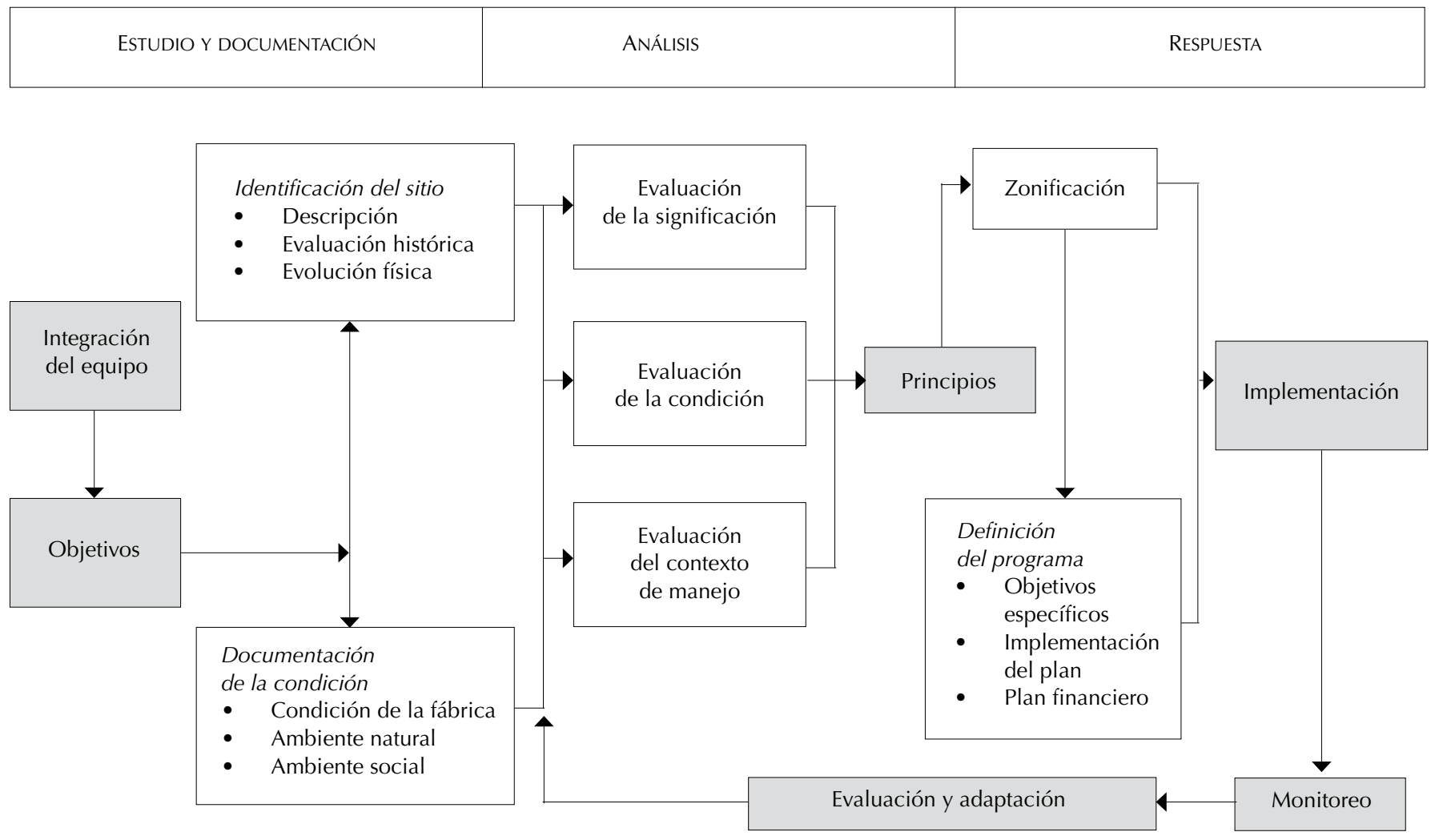

FIGURA 2. Proceso de planificación para el desarrollo del plan maestro de Chan Chan (Fuente: Castellanos 2001:112).

Propuesta de metodología para la mejora de los sistemas de diagnóstico como parte de la línea estratégica de mitigación del riesgo

La definición de una línea estratégica de mitigación del riesgo con un sistema de indicadores tiene como objeto realizar no sólo un diagnóstico, sino también un pronóstico de los escenarios posibles, para así priorizar los programas o planes de acción desde una perspectiva de prevención.

El diagnóstico se refiere al examen y análisis de la condición actual, para determinar las causas probables del deterioro. El pronóstico es implícito en el diagnóstico, pero no suele presentarse explícitamente. Al vincular las condiciones existentes con la documentación histórica y el diagnóstico, se pueden estimar las tasas de deterioro y, por lo tanto, evidenciar las prioridades de intervención para la toma de decisiones (Demas 2002:39, trad. de la autora).

Con base, primero, en el esquema de planificación desarrollado para la Zona Arqueológica de Chan Chan (Figura 2), que evidencia la importancia de la zonificación en el marco de la planificación estratégica; enseguida, en el modelo de Demas (2002), que incluye el análisis del contexto de manejo, y por último en el modelo desarroIlado por el MIBACT (2004), en el que la evaluación sistemática de los indicadores permite la adaptación del plan de manejo, en la Figura 3 se propone un esquema general con la finalidad de incorporar la línea estratégica de mitigación del riesgo con su sistema de indicadores, que también es aplicable a otras líneas estratégicas.

El riesgo se define como "la combinación de la probabilidad de que se produzca un evento y sus consecuencias negativas" (UNISDR 2009:29). Las ecuaciones para evaluar el riesgo $(R)$ consideran en general dos variables: la vulnerabilidad $(V)$ y las amenazas $(A)$, y existen variaciones en el concepto de la tercera variable, que ha sido definida como: los elementos en riesgo $(E R)$ (Varnes e IAEG 1984:10), la exposición (E) (Guevara et al. 2004:20) o el valor cultural (VC) (MIBACT 2004:15). Por lo tanto, el riesgo se define como:

$$
R=(V \times A)(E R ; E ; V C)
$$

Debido a que el valor de los elementos en riesgo, que constituye la tercera variable, debe definirse caso por caso, dependiendo de los valores otorgados por los involucrados con el sitio y manifestados en la declaración de significación, ${ }^{4}$ estos indicadores no se propondrán en el presente artículo. ${ }^{5}$

\footnotetext{
${ }^{4}$ Una declaración de significación proporciona un resumen conciso y esencial de la importancia cultural de un lugar (Australia ICOMOS 2013:5, trad. de la autora).

${ }^{5}$ Una referencia para su evaluación se puede encontrar en Díaz Fuentes (2016).
} 


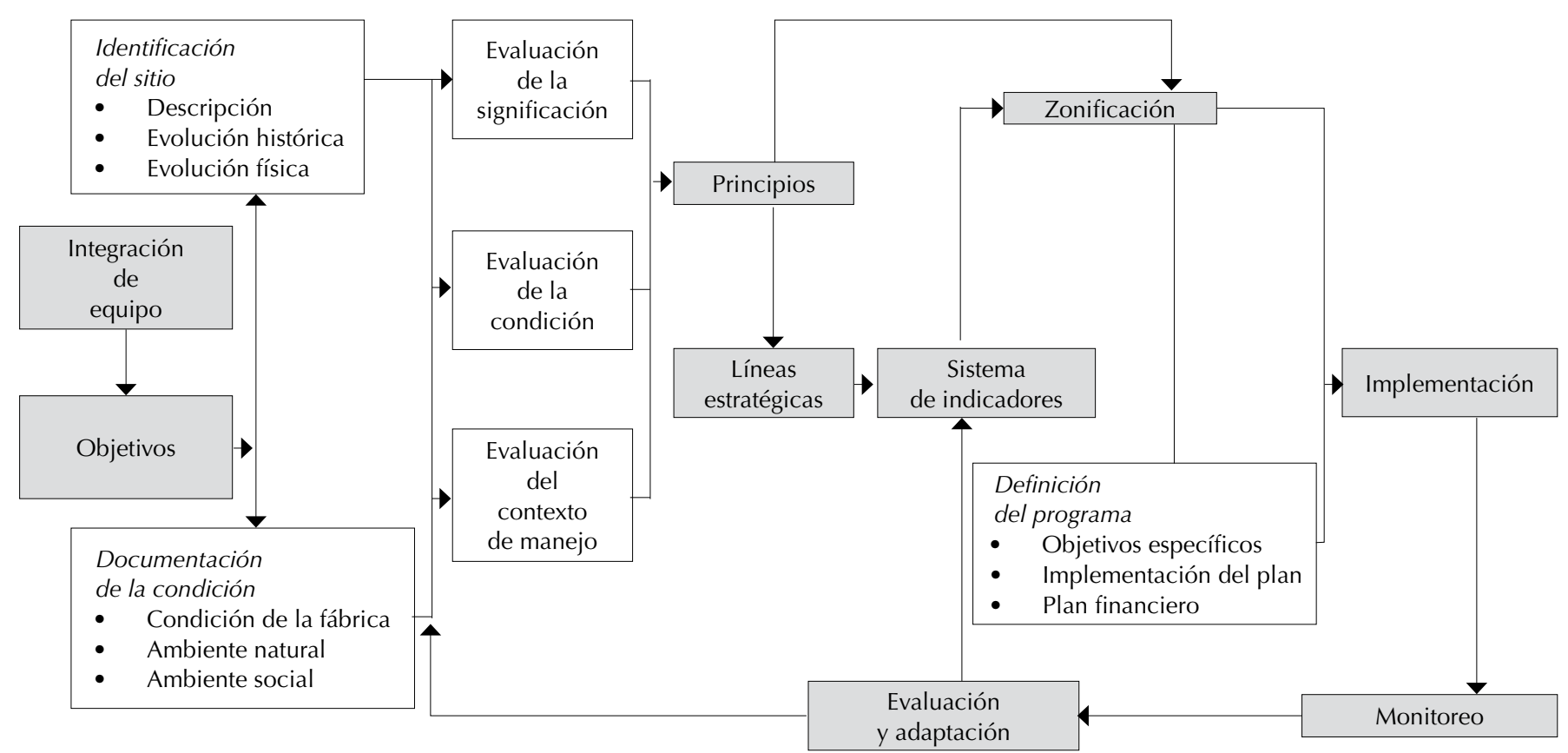

FIGURA 3. Propuesta de esquema general con el objeto de incorporar la línea estratégica de mitigación del riesgo con su sistema de indicadores (Fuente: Daniela Díaz 2017).

La metodología que se propone para mejorar los sistemas de diagnóstico del patrimonio inmueble como parte de la línea estratégica de mitigación del riesgo es la selección de un sistema de indicadores cualitativos y cuantitativos — que puedan reflejarse en una zonificación y monitorearse para la actualización del plan-, a partir de la revisión y aplicación de dos metodologías de evaluación del riesgo sísmico preexistentes y complementarias. Una es el método simplificado de evaluación de la vulnerabilidad sísmica de la tipología de iglesias, o LV1, establecido por el MIBACT en Italia (DPCM 2011:79-85), que analiza los posibles mecanismos de colapso que se activarían ante un evento sísmico; y la otra es la propuesta por Díaz Fuentes (2016) para la evaluación del riesgo en el patrimonio cultural inmueble, la cual consideró los conceptos desarrollados en los manuales internacionales para el manejo de los riesgos en el patrimonio cultural (Feilden 1987; Stovel 1998; ICCROM/UNESCO 2009; UNESCO/ICCROM/ICOMOS/IUCN 2010), que se centran en la identificación de las causas intrínsecas y extrínsecas de deterioro de los edificios históricos, entendidas como la vulnerabilidad y como las amenazas respectivamente.

\section{Método LV1 (DPCM 2011)}

La evaluación de la vulnerabilidad sísmica del patrimonio monumental se ha desarrollado extensamente en Italia debido a que el registro de los daños en los monumentos, luego de los recientes terremotos, ha permitido analizar estadísticamente los mecanismos de daño asociados a determinadas intensidades sísmicas, y proponer metodologías para evaluar su vulnerabilidad en una etapa preventiva; por esta razón, esta metodología forma parte del marco regulatorio del patrimonio italiano (DPCM 2011). Las iglesias, como tipología, son las más estudiadas en términos de su vulnerabilidad y probabilidad de daño ante un terremoto, ya que resultan dañadas incluso con sismos de baja intensidad, por lo que los registros de los daños son extensos (Lagomarsino y Podestà 2005). Con base en estos estudios, la guía italiana (DPCM 2011) propuso el método LV1 para definir un índice de vulnerabilidad en la tipología de iglesias, basado en el análisis de 28 mecanismos de colapso de sus macroelementos ${ }^{6}$ (Figura 4), que pueden activarse ante un fenómeno sísmico.

El índice de vulnerabilidad $\left(i_{v}\right)$ se calcula con la ecuación (1), donde $\mathrm{v}_{\mathrm{ki}}$ es el puntaje de los indicadores de fragilidad, $\mathbf{v}_{\mathrm{kp}}$ el puntaje de los dispositivos que aumentan la resistencia sísmica, y $\rho_{\mathrm{k}}$ el peso de cada mecanismo de colapso.

$$
i_{v}=\frac{1}{6} \frac{\sum_{k=1}^{28} \rho_{k}\left(v_{k i}-v_{k p}\right)}{\sum_{k=1}^{28} \rho_{k}}+\frac{1}{2}
$$

\footnotetext{
${ }^{6}$ Un macroelemento se entiende, en este caso, como una parte de la iglesia (en general, una pieza arquitectónica reconocible, como la fachada, el ábside-presbiterio, capillas, bóvedas, cúpulas, etc.) caracterizada por una respuesta sísmica en cierta medida autónoma del resto del edificio (Lagomarsino y Podestà 2005:57).
} 
ABACO DEI MECCANISMI DI COLLASSO DELLE CHIESE

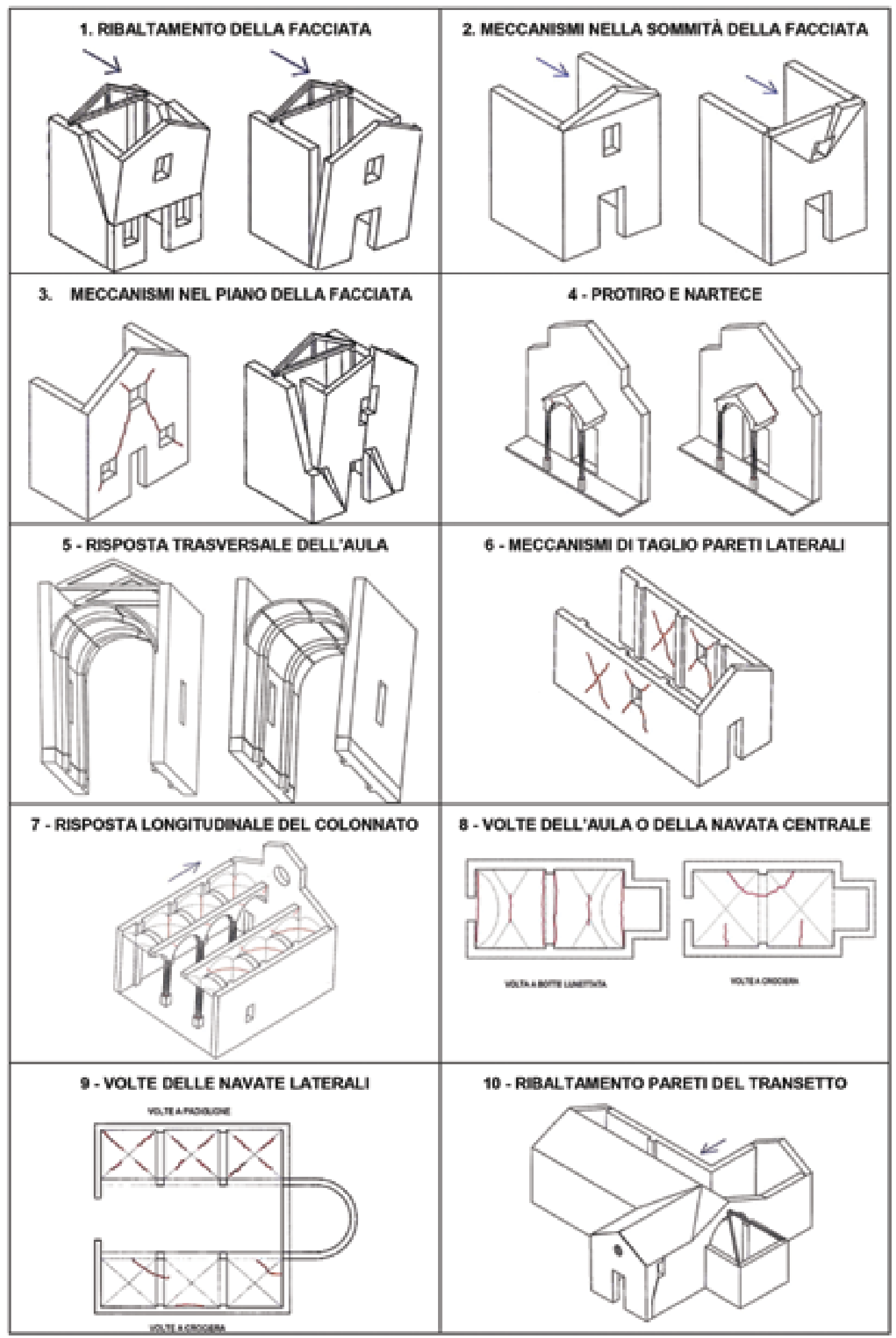

FIGURA 4. Selección de mecanismos de colapso por macroelemento (Fuente: MIBACT 2006). 
Por medio de este método es factible, a partir del índice de vulnerabilidad, calcular mediante las ecuaciones (2) y (3) la aceleración del suelo que genera el estado límite de daño (SLD) y el estado límite de salvaguardia de la vida (SLV). ${ }^{7}$ El índice de seguridad (IS) se calcula mediante la ecuación (4) dividiendo la aceleración correspondiente al estado límite por la aceleración máxima del suelo (AG o PGA, por sus siglas en inglés), siendo seguro si el resultado es mayor o igual a 1 (DPCM 2011:65).

$$
\begin{aligned}
& a_{S D D} S=0.025 \cdot 1.8^{2.75-3,44 c_{*}} \\
& a_{s, V} S=0.025 \cdot 1.8^{5.1-3.44 i_{*}} \\
& \text { IS }=a_{S L V} S \div a g_{S L V}
\end{aligned}
$$

\section{Método Díaz Fuentes (2016)}

Para evaluar la vulnerabilidad sísmica y las amenazas, el método simplificado Díaz Fuentes se aplica mediante dos herramientas. La que evalúa la vulnerabilidad se propuso con base en la ficha GNDT (Ferrini et al. 2003), la norma chilena núm. 3332 (INN 2013) para el patrimonio construido en tierra e investigaciones recientes sobre las construcciones de mampostería que abarcaron temas como: la estabilidad de las secciones de muros por la presencia de trabas transversales (Binda y Saisi 2001); la consolidación con materiales similares a los originales (cfr. Carocci 2001; Modena et al. 2009), y el comportamiento sísmico a través de los mecanismos de colapso (cfr. D'Ayala y Speranza 2002; Roca 2008; Díaz 2008). Además, se incluyeron algunos indicadores sociales que también podrían aumentar indirectamente la vulnerabilidad, como la accesibilidad, el aislamiento, la relación con el contexto geográfico y la comunidad, entre otros (Jorquera y AGR-CMN 2013). Esta herramienta evalúa indicadores (Figura 5) que se ponderan con un puntaje $(v)$ y con un peso $(p)$, relacionando con su importancia en el comportamiento sísmico del edificio (Díaz Fuentes 2016:189). El índice de vulnerabilidad (V) se calcula con la ecuación (5):

\footnotetext{
${ }^{7}$ El análisis de los estados límite tiene el propósito de identificar las diversas situaciones posteriores a un terremoto en que la estructura ya no cumple con los requisitos para los que se diseñó. Los estados límite que los lineamientos italianos consideran normalmente para el patrimonio cultural (DPCM 2011) son el de salvaguardia de vida (SLV), ya que garantiza no sólo la protección de los ocupantes, sino también la preservación del edificio, y el de daño (SLD), ya que asegura la posibilidad de uso del edificio después del terremoto (DPCM 2011:25, trad. de la autora).
}

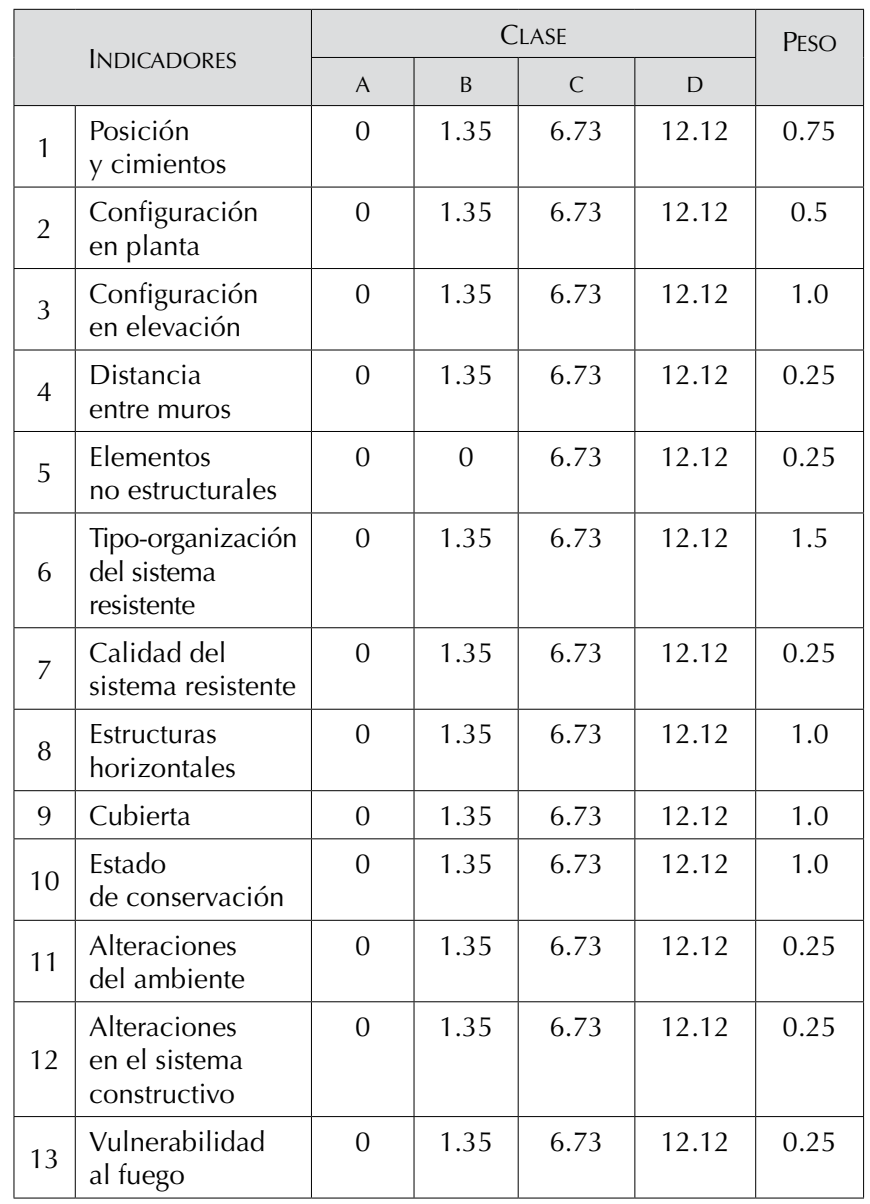

FIGURA 5. Puntaje y peso de los parámetros para definir el índice de vulnerabilidad (V) (Fuente: Díaz 2016:189).

$$
V_{j}=\sum_{i=1}^{n} v_{j,} p_{i}
$$

Por otra parte, la herramienta que evalúa las amenazas tomó en cuenta programas implementados, como la Carta del Rischio (ISCR 1992) en Italia, y documentos del ámbito de la planificación territorial desarrollados por el Centro Nacional de Prevención de Desastres (Cenapred) (cfr. Zepeda y González 2001; Ramos 2006) en México. Para definir el índice de amenaza sísmica se deben considerar sólo los indicadores propuestos en Díaz Fuentes (2016) que influyen en el comportamiento sísmico del edificio: la intensidad macrosísmica; el deslizamiento de laderas o fractura de la roca, y debido a que su principal consecuencia es el deterioro del material, procesos continuos como la erosión, el estrés físico, la contaminación atmosférica, la amenaza socio-organizacional y la falta de mantenimiento. El índice de amenaza $(A)$ se 
obtiene sumando todos los parámetros, considerando su influencia, como amplificador de la amenaza sísmica, en el comportamiento sísmico del edificio según la Figura 6 (Laterza et al. 2016a; 2016b; 2017).

\begin{tabular}{|l|l|c|c|c|}
\hline \multirow{2}{*}{\multicolumn{2}{|c|}{}} & \multicolumn{3}{c|}{ SEVERIDAD DEL DAÑO } \\
\cline { 3 - 5 } & $\begin{array}{c}\text { Sin } \\
\text { daño }\end{array}$ & $\begin{array}{c}\text { Leve o } \\
\text { gradual }\end{array}$ & Catastrófico \\
\hline \multirow{4}{*}{$\begin{array}{l}\text { Eventos } \\
\text { esporádicos }\end{array}$} & $\begin{array}{l}\text { Intensidad máx. } \\
\text { macro-sísmica }\end{array}$ & 0 & 0.20 & 0.40 \\
\cline { 2 - 5 } & $\begin{array}{l}\text { Deslizamiento } \\
\text { de ladera o fractura } \\
\text { de roca }\end{array}$ & 0 & 0.15 & 0.25 \\
\hline \multirow{3}{*}{$\begin{array}{l}\text { Procesos } \\
\text { continuos }\end{array}$} & Erosión & 0 & 0.05 & 0.10 \\
\cline { 2 - 5 } & Estrés físico & 0 & 0.05 & 0.10 \\
\cline { 2 - 5 } & Contaminación & 0 & 0.01 & 0.05 \\
\cline { 2 - 5 } & Socio-organizacional & 0 & 0.01 & 0.05 \\
\cline { 2 - 5 } & $\begin{array}{l}\text { Disminución } \\
\text { demográfica }\end{array}$ & 0 & 0.01 & 0.05 \\
\hline
\end{tabular}

FIGURA 6. Puntaje de los parámetros para definir el índice de amenaza sísmica (A) (Fuente: Laterza et al. 2016b:7).

El riesgo sísmico resultante $(R)$, ya definido como "la combinación entre la probabilidad de que ocurra un evento y sus consecuencias negativas" (UNISDR 2009), finalmente se calcula multiplicando el índice de vulnerabilidad sísmica $(V)$ por el índice de amenaza sísmica $(H)$, según la expresión (6):

$$
R s=V *(H+1)
$$

\section{Propuesta de sistema de indicadores}

Con base en los parámetros definidos en el método LV1, actualmente vigentes en los lineamientos que forman parte de la normativa italiana (DPCM 2011), y los definidos por Díaz Fuentes (2016) para evaluar las vulnerabilidades y amenazas, los indicadores que se propone incluir para la mejora del sistema de diagnóstico y aseguramiento de los inmuebles en los planes de manejo se dividen en dos grandes grupos: vulnerabilidad o causas de deterioro intrínsecas, y amenazas o causas extrínsecas (Figura 7).

Se proponen cinco tipos de indicadores de vulnerabilidad o causas de deterioro intrínsecas. El primero, mecanismos de colapso, se utiliza sólo cuando se analizan iglesias, y comprende los 28 mecanismos de colapso que eventualmente se activarán ante un terremoto, cuya vulnerabilidad se analiza con el método LV1 (DPCM 2011), obteniendo un índice de vulnerabilidad $\left(i_{v}\right)$ y un índice de seguridad (IS). El segundo, posición del edificio, comprende el análisis del emplazamiento del edificio en un terreno plano o en pendiente, en suelo blando o duro, y el tipo de cimentaciones. El tercero: inherente a la estructura, analiza el edificio en virtud de su configuración en planta y en elevación; la distancia entre muros, es decir, sus proporciones geométricas en función del espesor de aquéllos; los elementos no estructurales que pueden caer en caso de un terremoto; el tipo, organización y calidad de su sistema resistente, y sus estructuras horizontales y cubiertas. El cuarto, estado de conservación, comprende la evaluación de la condición actual del edificio y, además, las alteraciones negativas en su sistema constructivo y entorno. Finalmente, el quinto, vulnerabilidad al fuego, evalúa en términos generales la presencia de elementos que favorecerían la provocación o propagación de un incendio.

Asimismo, se proponen tres tipos de indicadores de amenazas o causas de deterioro extrínsecas, que se analizan con base en información histórica sobre escenarios de daño. El primero, amenazas naturales de acción ocasional, comprende la amenaza sísmica, de tsunami, volcánica, hidrometeorológica y de deslizamiento de laderas; el segundo, amenazas de naturaleza física, aquellas relacionadas con el ambiente, la erosión y el estrés físico, y el tercero, amenazas antrópicas, la amenaza química, la contaminación atmosférica, la amenaza socio-organizativa y la falta de mantenimiento. Como se explicó al describir el método propuesto por Díaz Fuentes (2016), al cuantificar los indicadores tanto de vulnerabilidad sísmica como de amenazas que influyen en el comportamiento sísmico del edificio, es posible calcular el riesgo sísmico total.

Aplicación de los indicadores cualitativos y cuantitativos del riesgo en las iglesias del sitio UNESCO I Sassi di Matera (Los Sassi de Matera)

En la revisión de antecedentes, como el registro de la Fundación La Scaletta (1960), los estudios de Tommaselli (2002) y el catastro de la municipalidad de Matera (CM 2014), entre otros, se encontraron 60 iglesias dentro del perímetro del sitio: 14 totalmente excavadas, 22 excavadas-construidas y 24 construidas. Luego de una primera caracterización arquitectónica, constructiva y del estado de conservación de los casos de estudio, los métodos simplificados LV1 (DPCM 2011) y Díaz Fuentes (2016) se aplicaron en seis iglesias (Laterza et al. 2017). Cuatro de éstas son de mampostería de piedra: Sant'Agostino y San Giovanni Battista, que presentan bóvedas de piedra en la techumbre, y San Francesco d'Assisi y San Pietro Caveoso, también con bóvedas de piedra y, además, techumbres de madera. Otras dos iglesias que se analizaron son: San Nicola dei Greci, que corresponde a la tipología completamente excavada en la roca, y Santa Maria de Armenis, principalmente excavada, pero cuya fachada fue construida en mampostería de piedra (Laterza et al. 2016b) (Figura 8). 


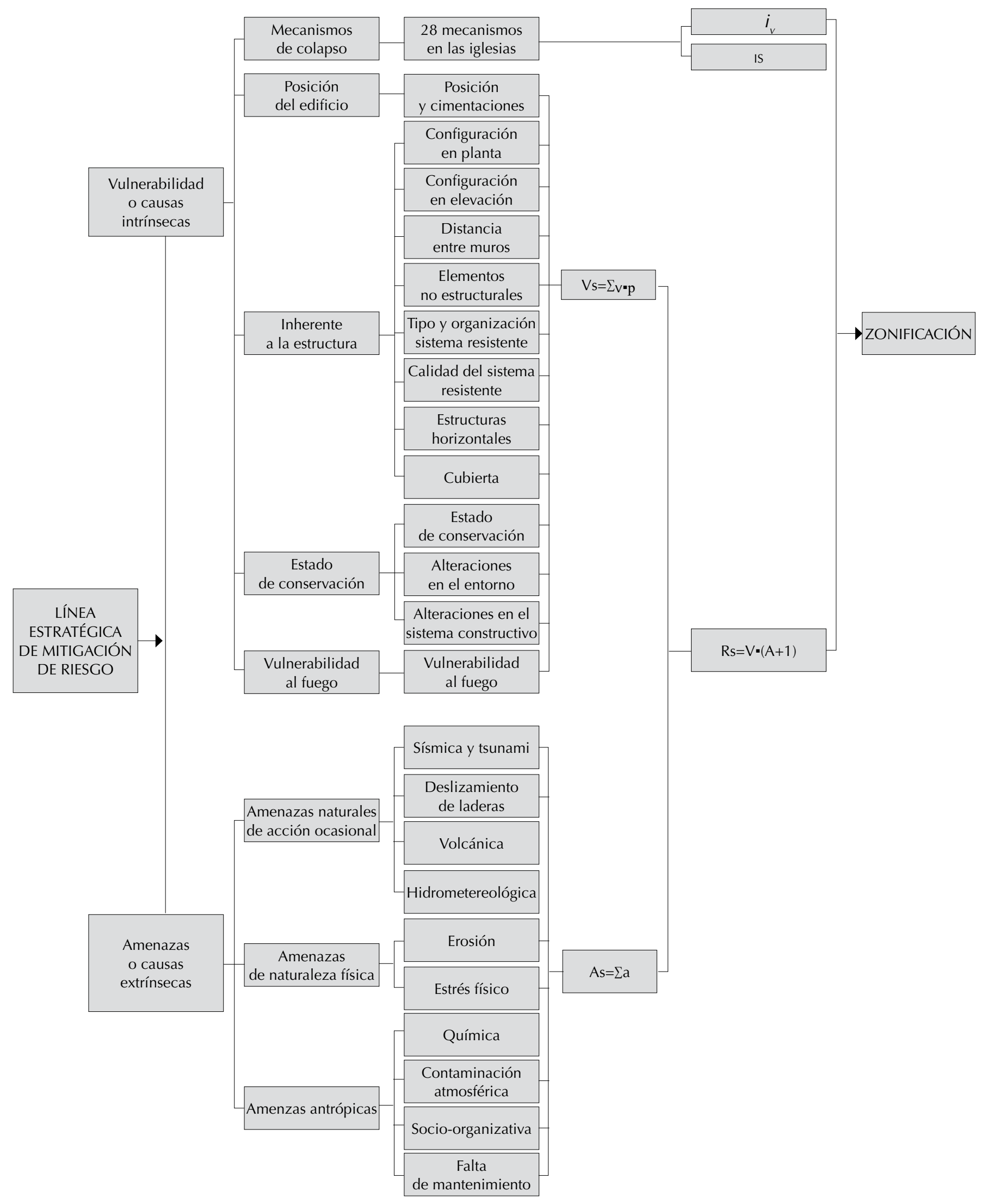

FIGURA 7. Propuesta de indicadores para incorporar la línea estratégica de mitigación del riesgo en los planes de manejo (Esquema: Daniela Díaz, 2017). 

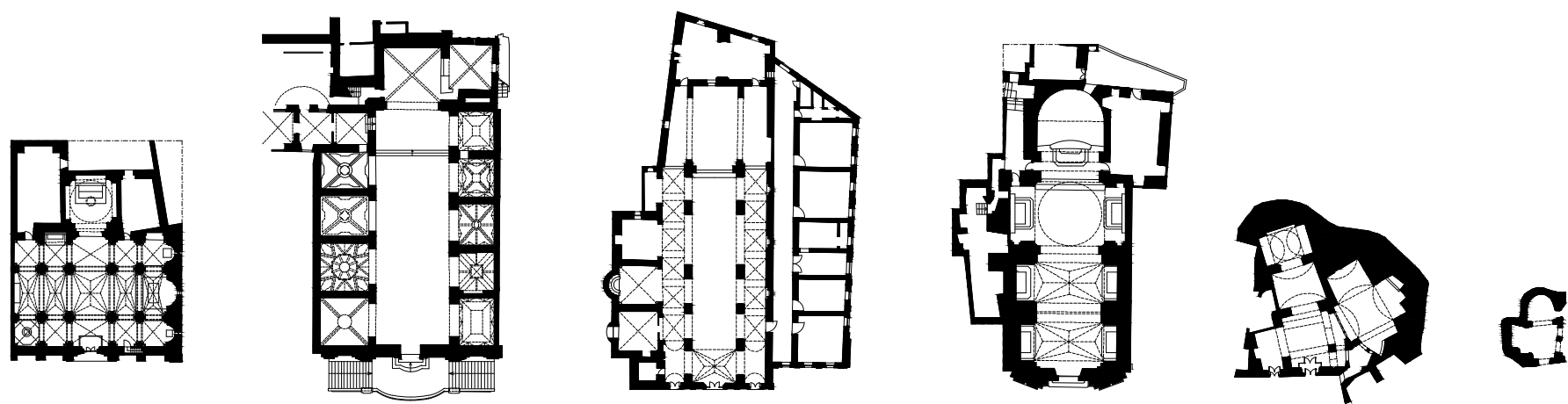

FIGURA 8. Planta de las iglesias (de izquierda a derecha): San Giovanni Battista, San Francesco d'Assisi, San Pietro Caveoso, Sant'Agostino, Santa Maria de Armenis y San Nicola dei Greci (Dibujo: Daniela Díaz y Marcella Chietera, 2016; fuentes: Planimetría de la Superintendencia de Arqueología, Bellas Artes y Paisaje de Italia [MIBACT 2016] y La Scaletta [1960]).

Con base en la aplicación de los indicadores para la evaluación del riesgo sísmico de las iglesias del sitio UNESCO I Sassi di Matera, fue posible determinar amenazas que afectan no sólo a las iglesias sino incluso a la totalidad del sitio. Si bien Matera se encuentra en una zona con sismicidad moderada, con una máxima intensidad macrosísmica observada igual a VII (Rovida et al. 2011), la ciudad presenta el más alto riesgo hidrogeológico de la región, debido a que se ubica aledaña al río encajonado Torrente Gravina, el cual genera prominentes barrancos formados por roca calcárea dolomítica dura, pero fracturada en capas y a menudo con deterioro kárstico, en una zona rodeada de fallas geológicas (Giasi 2006 [1999]). Por lo anterior, un posible programa debería abordar el estudio del riesgo de fractura de la roca en el borde del barranco del río Torrente Gravina, donde existen importantes monumentos, como la iglesia de San Pietro Caveoso y Sant'Agostino (Figura 9).

En relación con la aplicación de los indicadores de vulnerabilidad, el análisis cualitativo permitió realizar un diagnóstico que puede orientar programas específicos en el plan de manejo, como mejorar el arriostramiento de los muros laterales de las naves mediante contrafuertes; reforzar los frontones en las iglesias de San Francesco d'Assisi y San Giovanni Battista; compensar el empuje de las bóvedas con contrafuertes o tensores metálicos; eliminar la inyección de cemento sobre la bóveda de la iglesia de Sant'Agostino, y en los casos de las iglesias rupestres excavadas en la roca - San Nicola dei Greci y Santa Maria de Armenis-, proponer un proyecto para evitar la filtración de agua de lluvia, causante de su principal deterioro: la presencia de pátina biológica y el desgaste kárstico (Laterza et al. 2017). Si bien, como he dicho, la sismicidad de Matera es menor, según los resultados de la aplicación de los dos métodos (Figura 10) la iglesia de Sant'Agostino resultó en situación de inseguridad, por lo que se debe priorizar su conservación.

Por otra parte, los mecanismos de daño más probables que pueden activarse ante un terremoto son: aqueIlos fuera del plano y en el plano de la fachada; las fallas en las bóvedas de las naves central y laterales, presbiterio y ábside; el mecanismo fuera del plano en el ábside, y
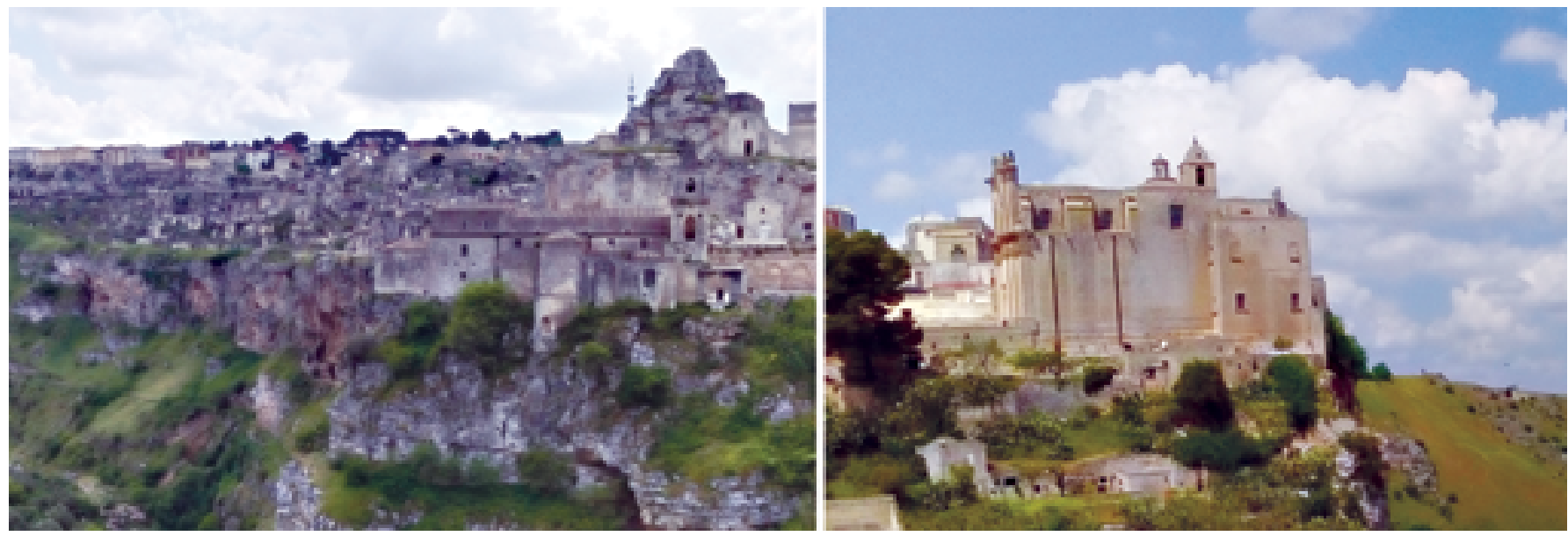

FIGURA 9. Ubicación de las iglesias de San Pietro (izquierda) y Sant'Agostino (derecha) en el borde del barranco del río Torrente Gravina (Fotografías: Daniela Díaz, 2016). 


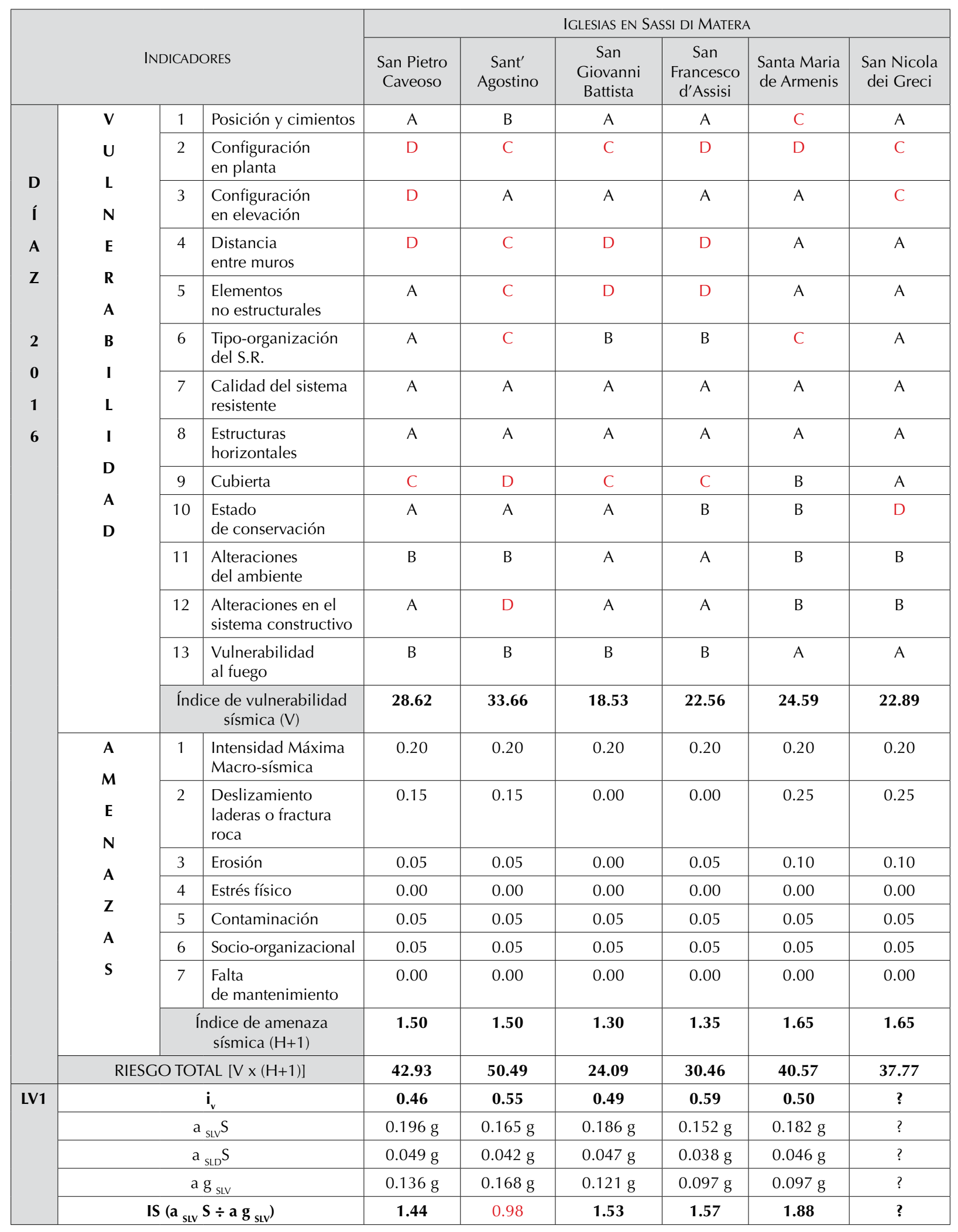

FIGURA 10. Resultados de la aplicación del método Díaz (2016) y del método LV1 (2011). Se destacaron en rojo los puntajes que evidencian los indicadores más vulnerables (Tabla: Daniela Díaz, 2017; fuente: Laterza et al. 2017). 
los mecanismos por la interacción de irregularidades en planta y en elevación. Por lo anterior, algunas medidas de conservación preventiva que debieran evaluarse en el marco de un proyecto de consolidación considerando los lineamientos italianos (DPCM 2011:104-121) son: la incorporación de tensores metálicos o contrafuertes para evitar los mecanismos fuera del plano de los muros y para compensar el empuje de las bóvedas, y bandas externas con materiales, como las fibras de carbono, para mejorar la conexión entre el frontón y el muro de fachada, y para consolidar las bóvedas.

\section{Conclusiones}

La implementación de políticas de prevención sísmica para el patrimonio cultural inmueble requiere conocimiento del riesgo al que están sometidos los edificios históricos a escala territorial. Para adquirirlo en un tiempo breve, y que sea homogéneo y específico sobre las vulnerabilidades y amenazas, pueden utilizarse métodos simplificados de diagnóstico del riesgo sísmico, como el Díaz Fuentes (2016) y el LV1 (DPCM 2011), cuyos parámetros permitirían aclarar un procedimiento de diagnóstico y aseguramiento de los edificios.

$\mathrm{Si}$ los indicadores propuestos en estos métodos se incorporan en los planes de manejo de los sitios inscritos en la Lista del Patrimonio Mundial de la UNESCO mediante una base de datos ligada a un sistema de información geográfica, cada edificio - en este caso, el patrimonio religioso- podría contar con una serie de datos estructurados a partir de fichas, relacionados con el conocimiento de la construcción, su estado de conservación, su vulnerabilidad sísmica y las amenazas inherentes. Además, con la posibilidad de cuantificar estos indicadores, se tendría un monitoreo sistemático de las modificaciones del riesgo de los inmuebles. Esta base de datos - en el marco de una estrategia de mitigación de riesgos preparada para el manejo del cambio- podría constituir una guía para la toma de decisiones y para definir programas específicos de conservación preventiva.

La utilidad de estos indicadores se pudo comprobar en la aplicación en el caso de estudio de las iglesias de Matera, ya que se identificaron amenazas, como la erosión kárstica del suelo, que requiere estudios minuciosos, y vulnerabilidades específicas de los edificios, que demandan obras de consolidación preventiva. Si bien el plan de acciones 2014-2019 del sitio inscrito en la Lista del Patrimonio Mundial de la UNESCO I Sassi di Matera propuso el desarrollo de un mapa de factores de riesgo ambiental (Colonna y Fiore 2014:11), hasta la fecha no se cuenta más que con uno general sobre la amenaza de fractura de la roca, basado en la carta geológica de la municipalidad de Matera (CM 2014), debido a que no se han propuesto indicadores específicos de evaluación del riesgo.

La incorporación de la línea estratégica de mitigación del riesgo con un sistema de indicadores evaluados me- diante dos metodologías simplificadas, como se ha propuesto en esta INVESTIGACIÓN, permitirá, por un lado, establecer listas de prioridades con base en el puntaje para, con ello, identificar los bienes más vulnerables, y además orientar las medidas de conservación preventiva, las obras de mitigación en el entorno de los bienes y la realización de estudios más detallados. Asimismo, el sistema con los indicadores propuestos podría facilitar la implementación y el monitoreo de la línea estratégica, aumentando así la sostenibilidad de los sitios.

La definición sistemática de una línea estratégica de mitigación del riesgo en los planes de manejo de los sitios de Patrimonio Mundial podría constituir un primer paso hacia la generación de compromisos políticos y técnicos entre los administradores de los sitios, las autoridades de gobierno y las instituciones encargadas de la protección del patrimonio, con el objeto de obtener recursos para la conservación preventiva con base en un diagnóstico homogéneo para todos los sitios. De esta forma, se respondería al requerimiento del Comité de Patrimonio Mundial de la UNESCO y, al mismo tiempo, se aumentaría la conciencia de los involucrados con los sitios sobre la necesidad de ejecutar acciones preventivas.

\section{Agradecimientos}

La investigación forma parte del Erasmus Mundus-Action 2, Partnerships Programme [EMA2] mediante el proyecto de beca y movilidad "Euro-Latin America partnership in natural risk mitigation and protection of the cultural heritage-ELARCH", financiado por la Comisión Europea y coordinado por la Universidad de la Basilicata, Italia. Este documento refleja sólo la opinión del autor, y la comisión no se hace responsable del uso que pueda hacerse de la información aquí contenida.

Agradezco a la estudiante de ingeniería civil y arquitectura Marcella Chietera su ayuda en la elaboración de la planimetría.

\section{Referencias}

\section{Australia ICOMOS}

2013 [1979] The Burra Charter: The Australia ICOMOS Charter for Places of Cultural Significance, Sidney, Australian National Committee of ICOMOS (Australia-ICOMOS), documento electrónico disponible en [http://australia.icomos.org/wp-content/uploads/The-Burra-Charter-2013-Adopted-31.10.2013. pdf], consultado en diciembre de 2017.

2013 Practice Note. Understanding and Assessing Cultural Significance, Version 1: November 2013, Sidney, Australian National Committee of ICOMOS (Australia-ICOMOS), documento electrónico disponible en [http://australia.icomos.org/wp-content/uploads/Practice-Note_Understanding-and-assessing-cultural-significance.pdf], consultado en diciembre de 2017.

ACH-FCH CDMX

2011 Plan Integral de Manejo del Centro Histórico de la Ciu- 
dad de México (2011-2016), México, Autoridad del Centro Histórico, Fideicomiso Centro Histórico de la Ciudad de México (ACH-FCH CDMX).

Badia, Francesco

2012 "Monitoraggio e controllo della gestione dei siti UNESCO. Il piano di gestione come opportunità mancata?", Tafter Journal (52), documento electrónico disponible en [http://www. tafterjournal.it/2012/10/01/monitoraggio-e-controllo-dellagestione-dei-siti-unesco-il-piano-di-gestione-come-opportunita-mancata], consultado en marzo de 2017.

Binda, Luigia y Antonella Saisi

2001 "State of the art of research on historic structures in Italy", Advanced Research Centre for Cultural Heritage Interdisciplinary Projects (ARCCHIP), documento electrónico disponible en [http://www.arcchip.cz/w11/w11_binda.pdf], consultado en mayo de 2015.

Carocci, Caterina

2001 "Guidelines for the Safety and Preservation of Historical Centres in Seismic Areas", en Paulo B. Lourenço y Pere Roca (eds.), Historical Constructions 2001. Possibilities of Numerical and Experimental Techniques. Proceedings of the 3rd International Seminar, Guimaraes, Portugal, University of Minho, November 7-8-9, 2001, Guimarães, Universidade do Minho, 145-166, documento electrónico disponible en [http://www.hms.civil.uminho.pt/sahc/2001/page\%20145166\%20_Carocci_.pdf], consultado en mayo de 2015.

Castellanos, Carolina

2001 "Sustainable management for archaeological sites: the case of Chan Chan, Peru", en Jeanne Marie Teutonico y Frank Matero (eds.), Managing Change: Sustainable Approaches to the Conservation of the Built Environment. 4th Annual US/ ICOMOS International Symposium Organized by US/ICOMOS, the Graduate Program in Historic Preservation of the University of Pennsylvania, and the Getty Conservation Institute Philadelphia, Pennsylvania, April 2001, Los Ángeles, Getty Conservation Institute (GCI) (Serie Proceedings), 107-116.

Castellanos, Carolina, Françoise Descamps y María Isaura Aráuz 2002 "Plan de manejo Joya de Cerén, El Salvador", Consejo Nacional para la Cultura y el Arte, Getty Conservation Institute, documento electrónico disponible en [https://www.getty. edu/conservation/publications_resources/pdf_publications/ pdf/plan_de_manejo.pdf], consultado en diciembre de 2017. $\mathrm{CM}$

2014 "Planimetria Rioni Sassi, su base aerofotogrammetrica eseguita nel 1989", Matera, Settore Gestione del Territorio Ufficio Sassi, Comune di Matera (CM).

Colonna, A. y D. Fiore

2014 I Sassi e il parco delle chiese rupestri di Matera Patrimonio della'Umanità. Piano di gestione 2014-2019. Technical Report, Matera, Italia.

D'Ayala, Dina y Elena Speranza

2002 "An Integrated Procedure for the Assessment of Seismic Vulnerability of Historic Buildings", en 12th European Conference on Earthquake Engineering, Londres, Elsevier Science, 1-10.

Darvill, T. y A. Fulton

1998 MARS: The Monuments at Risk Survey of England, 1995:
Main Report, Bournemouth, Bournemouth University/English Heritage.

Demas, Martha

2002 "Planning for Conservation and Management of Archaeological Sites. A Values-based Approach", en Jeanne Marie Teutonico y Gaetano Palumbo (eds.), Management Planning for Archaeological Sites. An International Workshop Organized by the Getty Conservation Institute and Loyola Marymount University, May 19-22, 2000, Corinth, Greece, Los Ángeles, Getty Conservation Institute (Serie Proceedings), 27-54.

Díaz, César

2008 "Lesiones estructurales en los edificios de la arquitectura tradicional mediterránea", en X. Casanovas (dir.), Método Rehabimed, arquitectura tradicional mediterránea, vol. II, Barcelona, Consorcio Rehabimed, 195-208.

Díaz Fuentes, Daniela

2016 Diseño de herramientas de evaluación del riesgo para la conservación del patrimonio cultural inmueble. Aplicación en dos casos de estudio del norte andino chileno, México, Escuela Nacional de Conservación, Restauración y Museología/Instituto Nacional de Antropología e Historia.

DPCM

2011 "Linee guida per la valutazione e riduzione del rischio sismico del patrimonio culturale-allineamento alle nuove Norme tecniche per le costruzioni", Gazzetta Ufficiale della Repubblica Italiana, Direttiva del Presidente del Consiglio dei Ministri (DPCM), núm. 47, 26 de febrero, documento electrónico disponible en [http://www.beniculturali.it/mibac/export/MiBAC/sito-MiBAC/Contenuti/Avvisi/visualizza_asset. html_1141304737.html], consultado en diciembre de 2017. ELARCH

2016 Euro-Latin American Partnership in Natural Risk Mitigation and Protection of the Cultural Heritage, Euro-Latin America partnership in natural Risk mitigation and protection of the Cultural Heritage (ELARCH), documento electrónico [página web] disponible en [www.elarch.org], consultado en noviembre de 2016.

Espiñeira, María Eugenia y María Elena Noël

2004 "Plan de manejo de las Oficinas Salitreras Humberstone y Santa Laura 2004-2009", documento inédito, Santiago, Consejo de Monumentos Nacionales, Dirección de Arquitectura, Ministerio de Obras Públicas, Corporación Museo del Salitre.

Feilden, B. M.

1987 Between Two Earthquakes: Cultural Property in Seismic Zones, Roma, International Centre for the Study of the Preservation and Restoration of Cultural Property/Getty Conservation Institute.

Ferrini, M., A. Melozzi, A. Pagliazzi y S. Scarparolo (coords.) 2003 Rilevamento della vulnerabilità sismica degli edifici in muratura. Manuale per la compilazione della Scheda GNDT/ CNR di Il livello, Roma, Direzione Generale delle Politiche Territoriale e Ambientali, Settore Servizio Sismico Regionale, documento electrónico disponible en [http://www.regione.toscana.it/documents/10180/12262198/vsm_man. 
pdf/095d3648-191d-43aa-ae88-ad78cff79fb3], consultado en diciembre de 2017.

Fundación La Scaletta

1960 Le chiese rupestri di Matera, Roma, La Scaletta/De Luca Editori.

Giasi, N.

2006 [1999] "Studio geologico. Carta geologica e della stabilità dei versanti", Piano Regolatore Generale. Variante Matera, gráfico [plano], All. 2 B, Comune di Matera.

Guevara Ortiz, Enrique, Roberto Quaas Weppen y Georgina Fernández Villagómez

2004 "Lineamientos generales para la elaboración de atlas de riesgos", en Violeta Ramos Radilla (coord.), Guía básica para la elaboración de atlas estatales y municipales de peligros y riesgos, México, Secretaría de Gobernación/Centro Nacional de Prevención de Desastres (Serie Atlas Nacional de Riesgos), 15-20.

Guido, Manuel Roberto (coord.)

2002 Le città tardo barocche del Val di Noto (Sicilia sudorientale). Piano di gestione, Ministero per i Beni e per le Attività Culturali, documento electrónico disponible en [http:// www.comune.noto.sr.it/files/noto/images/stories/cultura/ unesco/appendice\%20a.pdf], consultado en marzo de 2017.

Hoyle, Ana María y Carolina Castellanos

1999 Plan Maestro para la Conservación y Manejo del Complejo Arqueológico Chan Chan, Lima, Dirección General de Patrimonio Arqueológico, Instituto Nacional de Cultura.

$\mathrm{HS} / \mathrm{CEC} / \mathrm{EWT}$

2011 The Old and New Towns of Edinburgh World Heritage Site. Management plan 2011-2016, Historic Scotland, City of Edinburgh Council, Edinburgh World Heritage (HS/CEC/EWT), documento electrónico disponible en [http://www.ewht.org. uk/uploads/downloads/WHS_Management_Plan\%202011. pdf], consultado en marzo de 2017.

ICCROM/UNESCO

2009 Manual de gestión de riesgos de colecciones, Partnership for the Preventive Conservation of Endangered Museum Collections in Developing Countries, Centre for the Study of the Preservation and Restoration of Cultural Property/ United Nations Educational, Scientific and Cultural Organization (ICCROM/UNESCO), documento electrónico disponible en [http:// unesdoc.unesco.org/images/0018/001862/186240s.pdf], consultado en mayo de 2015.

ICOMOS

2000 "Informe mundial 2000 de ICOMOS sobre monumentos y sitios en peligro", H@R!: Heritage at Risk/Patrimonio en Peligro, International Council on Monuments and Sites (ICOMOS), documento electrónico disponible en [https://www. icomos.org/risk/world_report/2000/intro_spa.htm], consultado en marzo de 2017.

INGV

2017 Centro Nazionale Terremoti, Istituto Nazionale di Geofisica e Vulcanologia (INGV), documento electrónico [página web] disponible en [http://cnt.rm.ingv.it], consultado en noviembre de 2016.
INAH-Conaculta

2009 "Plan de manejo Zona de Monumentos Arqueológicos Chichén Itzá", documento inédito, México, Instituto Nacional de Antropología e Historia, Consejo Nacional para la Cultura y las Artes (INAH-Conaculta).

INN

2013 Norma chilena núm. 3332, oficializada el año 2013: Estructuras-intervención de construcciones patrimoniales de tierra cruda. Requisitos del proyecto estructural, Santiago, Instituto Nacional de Normalización (INN). ISCR

1992 Carta del Rischio, Istituto Superiore per la Conservazione ed il Restauro (ISCR), documento electrónico [página web] disponible en [http://www.cartadelrischio.it/index.asp], consultado en noviembre de 2016.

Johnston, Chris

2010 Conservation Management Plans: Managing Heritage Places. A Guide, Melbourne, Heritage Council of Victoria.

Jorquera, Natalia y AGR-CMN

2013 Fichas para la evaluación de los riesgos en los monumentos nacionales de Chile, Santiago, Natalia Jorquera y Área de Gestión del Riesgo, Consejo de Monumentos Nacionales (AGR-CMN), documentos inéditos, Centro de Documentación, Consejo de Monumentos Nacionales.

Kerr, James Semple 2013 [1996] The Conservation Plan: A Guide to the Preparation of Conservation Plans for Places of European Cultural Significance, Sydney, Australian National Committee of ICOMOS (Australia-ICOMOS), documento electrónico disponible en [http://australia.icomos.org/publications/the-conservation-plan], consultado en septiembre de 2017.

Labadi, Sophia

2015 UNESCO, Cultural Heritage, And Outstanding Universal Value. Value-Based Analyses Of The World Heritage And Intangible Cultural Heritage Conventions, Lanham, AltaMira Press (Serie Archaeology in Society).

Lagomarsino, Sergio y Stefano Podestà

2005 "Inventario e vulnerabilità del patrimonio monumentale dei parchi dell'Italia centro-meridionale, vol. III-Analisi di vulnerabilità e rischio degli edifici monumentali", L'Aquila, Istituto Nazionale di Geofisica e Vulcanologia, Gruppo Nazionale per la Difesa dai Terremoti, documento electrónico disponible en [https://emidius.mi.ingv.it/GNDT2/Att_scient/ Prodotti_consegnati/Dolce_Zuccaro/Task3/SAVE_Task3.pdf], consultado en diciembre de 2017.

Laterza, Michelangelo, Antonella Guida, Michele D'Amato, Antonello Pagliuca, Daniela Díaz y Marcella Chietera

2016a "Technical and technological qualification of ancient buildings. The case of churches in Sassi di Matera", ponencia presentada en la XII International Conference on Structural Repair and Rehabilitation, CINPAR, 26-29 de octubre, Portugal, Faculdade de Engenharia da Universidade do Porto.

Laterza, Michelangelo, Michele D’Amato y Daniela Díaz 2016b "Ancient masonry cathedrals in Matera landscape: seismic assessment and risk mitigation", ponencia presentada en CINPAR 2016. XII International Conference on Structural 
Repair and Rehabilitation, 26-29 de octubre, Oporto, Faculdade de Engenharia da Universidade do Porto.

2017 "Caracterización técnica y evaluación sísmica de edificios históricos: el caso de las iglesias en Sassi di Matera", artículo y cartel presentados en el XXXI Salón Tecnológico de la Construcción EXCO 2017, 20-24 de febrero, Valencia, Feria Internacional CEVISAMA 2017.

Medina-González, Isabel

2006 “Una aproximación a la gestión y planificación estratégica en zonas arqueológicas: el caso de México", Anuario Turismo y Sociedad, Universidad Externado de Colombia, VII:54-64.

MIBACT

2004 Il modello del piano di gestione dei beni culturali iscritti alla lista del Patrimonio dell'Umanità. Linee Guida, Commissione Nazionale Siti UNESCO e Sistemi Turistici Locali, Ministero per i Beni e le Attività Culturali e del Turismo (MIBACT), documento electrónico disponible en [www.unesco.beniculturali.it/getFile.php?id=44], consultado en marzo de 2017. 2006 Scheda per il rilievo del danno ai beni culturali-Chiese. Modello A-DC PCM-DPC, documento electrónico disponible en [http://www.beniculturali.it/mibac/multimedia/MiBAC/ documents/1338454237471_allegato4.pdf], consultado en noviembre de 2016.

2016 "Planimetria delle chiese di San Giovanni Battista, San Francesco d'Assisi, San Pietro Caveoso e Sant'Agostino", Ministero per i Beni e le Attività Cculturali e del Turismo (MIBACT), gráfico [plano], Archivo de la Soprintendenza Archeologia, Belle Arti e Paesaggio de Matera.

Modena, Claudio, Filippo Casarin, Francesca do Porto, Enrico Garbin, Nicola Mazzon, Marco Munari, Matteo Panizza y Maria Rosa Valluzzi

2009 "Structural Interventions on Historical Masonry Buildings: Review of Eurocode 8 Provisions in the light of the Italian experience", en E. Cosenza (ed.), Eurocode 8 Perspectives from the Italian Standpoint Workshop, Nápoles, Doppiavoce, 225-236.

Podestà, Stefano, Chiara Romano y Lorenzo Scandolo 2010 "Molise, valutazione della sicurezza sismica a livello territoriale", en VV. AA., Linee guida per la valutazione e riduzione del rischio sismico del patrimonio culturale allineate alle nuove Norme tecniche per le costruzioni (d. m. 14 gennaio 2008), Roma, Ministero per i Beni e le Attività Culturali e del Turismo, 324-343, documento electrónico disponible en [http://www.beniculturali.it/mibac/multimedia/MiBAC/documents/1295445079370_4)Esempi(6).pdf], consultado en diciembre de 2017.

Ramos Radilla, Violeta (coord.)

2004 Guía básica para la elaboración de atlas estatales y municipales de peligros y riesgos, México, Secretaría de Gobernación/Centro Nacional de Prevención de Desastres (Serie Atlas Nacional de Riesgos).

2006 Guía básica para la elaboración de atlas estatales y municipales de peligros y riesgos. Conceptos básicos sobre peligros, riesgos y su representación geográfica, México, Secre- taría de Gobernación/Centro Nacional de Prevención de Desastres (Serie Atlas Nacional de Riesgos).

Roca, Pere

2008 "El comportamiento sísmico de las construcciones tradicionales de paredes de obra de fábrica", en X. Casanovas (dir.), Método Rehabimed. Arquitectura tradicional mediterránea, vol. II, Barcelona, Consorcio Rehabimed, 216-225.

Rovida, A., R. Camassi, P. Gasperini y M. Stucchi (coord.)

2011 Catalogo parametrico dei terremoti italiani, Milán, Istituto Nazionale di Geofisica e Vulcanologia, documento electrónico [página web] disponible en [http://emidius.mi.ingv.it/ CPTI], consultado en marzo de 2017.

Stovel, Herb

1998 Risk Preparedness: A Management Manual for World Cultural Heritage, Roma, International Centre for the Study of the Preservation and Restoration of Cultural Property/United Nations Educational, Scientific and Cultural Organization/ World Heritage Centre/International Council on Monuments and Sites.

Tommaselli, Mario

2002 Chiese rupestri di Matera e del suo territorio, Lecce, Capone Editore.

UNESCO

1972 "Convention concerning the Protection of the World Cultural and Natural Heritage. Adopted by the General Conference at its seventeenth session Paris, 16 november 1972", United Nations Educational, Scientific and Cultural Organisation (UNESCO), documento electrónico disponible en [http://whc. unesco.org/en/conventiontext], consultado en marzo de 2017. 2017 "Lista de Patrimonio Mundial", United Nations Educational, Scientific and Cultural Organisation (UNESCO), documento electrónico [página web] disponible en [http://whc. unesco.org/es/list], consultado en marzo de 2017.

UNESCO-WHC

2015 Operational Guidelines for the Implementation of the World Heritage Convention, París, United Nations Educational, Scientific and Cultural Organisation, World Heritage Centre (UNESCO-WHC), documento electrónico disponible en [http://whc.unesco.org/en/guidelines], consultado en diciembre de 2017.

UNESCO/ICCROM/ICOMOS/IUCN

2010 Managing Disaster Risks for World Heritage, París, United Nations Educational, Scientific and Cultural Organisation/World Heritage Centre/ International Centre for the Study of the Preservation and Restoration of Cultural Property/ International Council on Monuments and Sites/International Union for Conservation of Nature (UNESCO/ICCROM/ICOMOS/IUCN), documento electrónico disponible en [http://whc. unesco.org/en/managing-disaster-risks], consultado en diciembre de 2017.

2013 Managing Cultural World Heritage, París, United Nations Educational, Scientific and Cultural Organisation, World Heritage Centre/International Centre for the Study of the Preservation and Restoration of Cultural Property/International Council on Monuments and Sites/International Union 
for Conservation of Nature (UNESCO/ICCROM/ICOMOS/IUCN), documento electrónico disponible en [http://openarchive. icomos.org/1465], consultado en diciembre de 2017.

UNISDR

2009 Terminología sobre Reducción del Riesgo de Desastres, Ginebra, United Nations International Strategy for Disaster Reduction (UNISDR), documento electrónico disponible en [http://www.preventionweb.net/files/7817_UNISDRTerminologySpanish.pdf], consultado en octubre de 2017.

Varnes, David J. e IAEG

1984 Landslide Hazard Zonation: A Review of Principles and Practice, París, United Nations Educational, Scientific and Cultural Organization.

Young, Christopher

2002 "Hadrian's Wall, United Kingdom", en Jeanne Marie Teutonico y Gaetano Palumbo (eds.), Management Planning for Archaeological Sites. An International Workshop Organized by the Getty Conservation Institute and Loyola Marymount University, 19-22 May 2000, Corinth, Greece, Los Ángeles, Getty Conservation Institute (Serie Proceedings), 60-67.

Young, Christopher, Amanda Chadburn e Isabelle Bedu 2009 Stonehenge World Heritage Site Management Plan 2009, Londres, English Heritage.

Zepeda Ramos, Óscar y Susana González Martínez (eds.) 2001 Diagnóstico de peligros e identificación de riesgos de desastres en México. Atlas Nacional de Riesgos de la República de México, México, Secretaría de Gobernación/Centro Nacional de Prevención de Desastres.

\section{Síntesis curricular del/los autor/es}

\section{Daniela Andrea Díaz Fuentes}

Università degli Studi della Basilicata (Unibas), Italia daniela.diazfue@gmail.com

Arquitecta (Universidad de Chile [UCH]) y maestra en conservación y restauración de bienes culturales inmuebles (Escuela Nacional de Conservación, Restauración y Museología, Instituto Nacional de Antropología e Historia [ENCRyM-INAH], México). Trabajó en el Consejo de Monumentos Nacionales de Chile. Actualmente, cursa estudios de doctorado (Dipartimento delle Culture Europee e del Mediterraneo [DICEM], Departamento de Culturas del Mediterráneo, Università degli Studi della Basilicata [Unibas], Universidad de la Basilicata Matera, Italia). En el 2015 fue galardonada por el Premio Haz Tu Tesis en Cultura, otorgado por el Consejo Nacional de la Cultura y las Artes (CNCA) de Chile. En el 2016 recibió el Premio INAH a la mejor tesis de maestría en conservación-restauración de monumentos.

Postulado/Submitted: 21.03.2017

Aceptado/Accepted: 22.01.2018

Publicado/Published: 28.02.2018 